\title{
Transfer Printing Enabled Soft Composite Films for Tunable Surface Topography
}

\author{
Zining Yang ${ }^{\mathrm{a}}$, Qianli Chen ${ }^{\mathrm{b}}$, Ahmed E. Elbanna ${ }^{\mathrm{b}}$, Seok Kim ${ }^{\mathrm{a} *}$ \\ ${ }^{a}$ Department of Mechanical Science and Engineering, University of Illinois at Urbana Champaign, United States \\ ${ }^{b}$ Department of Civil and Environmental Engineering, University of Illinois at Urbana Champaign, United States
}

\section{HIGHLIGHTS}

- Soft composite films are fabricated by transfer printing silicon ribbons onto elastomeric films in a spatially organized manner.

- Tension-induced corrugation on the soft composite film is experimentally observed.

- Corrugation amplitude in response to applied stretch is characterized.

- Mechanism of corrugation formation on the composite film is elucidated by transformation rule.

- Effect of design parameters on corrugation is experimentally and numerically investigated.

\section{ARTICLE INFO}

Article history:

Keywords:

Transfer printing

Tunable surface

Soft composite

\section{GRAPHICAL ABSTRACT}

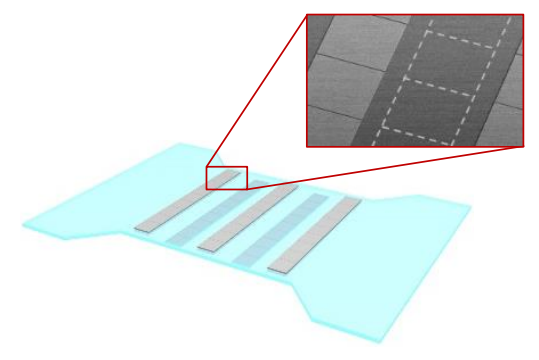

Stretch-free State

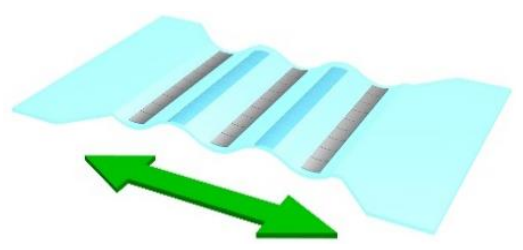

Stretched State

\begin{abstract}
Transfer printing, an emerging manufacturing technique for heterogeneous material integration, is combined with the unique mechanics of soft composite films to realize a responsive surface for tunable topography in this work. Soft composite films are fabricated by transfer printing thin silicon ribbons on elastomeric films in a staggered manner. The corrugation formation in soft composite films requires a well-organized spatial distribution of the silicon ribbons which is enabled by transfer printing processes developed here. The corrugation development in response to applied stretching is characterized by optical metrology and the results fit with the numerical model to a good extent. The experimental results reveal that the maximum corrugation amplitude of the film can go beyond $25 \%$ of the total film thickness. Furthermore, the effect of geometrical and material parameters on corrugation formation is investigated experimentally and numerically. The results shown here suggest an alternative way to induce tunable surface topography.
\end{abstract}

\section{Introduction}

Surface corrugation is commonly manifested in daily life such as in water waves, cloud formations, sand dunes, wrinkles on human skin as well as in corrugated cardboard and metal roof panels. Inspired by such examples, developing engineered soft materials to achieve tunable surface topography, especially wavy geometry, have led to a wide range of applications such as responsive surface with tunable dry adhesion and wettability [1-3], novel electronic devices [4,5], diffraction gratings [6], microlenses [7] and tunable metamaterials [8]. For most of these applications, the soft materials are manufactured into the form of thin films and the corrugation patterns can

* Corresponding author. Tel.: +1-217-265-5656 ; fax: +1-217-244-9956.

E-mail address: skm@illinois.edu xxxx-xxxx/\$ - see front matter $\odot 2013$ xxxxxxxx. Hosting by Elsevier B.V. All rights reserved. http://dx.doi.org/10.1016/j.rgo.2013.10.012 
appear/reappear in response to external forces. Certain mechanical effects are exploited for the formation of corrugation and up to now the most well-established mechanism is to utilize the buckling phenomenon. Yang et al. reviewed the two common routes to engender buckling on the surface of an elastomeric material, namely by swelling a confined substrate or by pre-straining the substrate of a bilayer system [9]. In the first case, a polymer (e.g., hydrogel) film is initially confined on a rigid substrate and subsequently swelled by solvent. The surface has a tendency to expand and build up a compressive strain since the bottom surface of the polymer is fixed. In the second case, a thin coating is applied on a pre-strained soft substrate and compressive strain is spontaneously generated in the coating upon the releasing of the prestrain. In both cases the outer surface of the specimens are compressively strained such that buckling occurs and wrinkling patterns are formed as a result. In addition to inelastic buckling, other mechanical effects are also exploited for corrugation generation. Recently, Chen et al. numerically investigated the elastic response of a twophase composite film that consists of hard inclusions and soft matrix under externally applied concentric tension. They found that by careful design of the inclusion pattern, it is possible to induce corrugation normal to the direction of stretch [10]. The results suggest an alternative mechanism to realize tunable surface topography and could potentially open up new applications such as tunable band gap formation and tunable surface roughness development. Nevertheless, their numerical prediction for two-phase composites has not been validated experimentally. To this end, a large-scale manufacturing process which enables the integration of hard inclusion into a soft film in a spatially organized manner needs to be developed.

Transfer printing is an emerging technique for heterogeneous material integration [11-13]. In the regime of deterministic micro-transfer printing [13], microscale objects (also referred to as inks) are prepared on a donor substrate by microfabrication. An elastomeric transfer element (also referred to as a stamp) is brought into contact with the inks on the donor substrate with high preload. Fast separation between the stamp and donor substrate enhances the adhesion at the ink/stamp interface due to viscoelastic effect of the stamp, resulting in the fracture at ink/donor interface as the inks stay with the stamp [14]. Those inks can be further relayed to another substrate (also referred to as a receiving substrate) if necessary. Transfer printing, in conjunction with other manufacturing techniques (e.g., molding, microfabrication, self-assembly, etc.), provides an approach to integrate disparate classes of materials into one unique system since different materials are prepared on their own substrates with optimal methods and assembled afterwards.

In this work, we utilize the emerging transfer printing technique not only to realize soft composite films exhibiting tunable surface topography but also to experimentally validate the mechanics of tension-induced corrugation. The geometry and materials of the composite film are carefully selected in order to maximize the corrugation amplitude. The manufacturing process is demonstrated in detail and the corrugation is characterized in both qualitative and quantitative manners. Control samples with varying design parameters including different soft film modulus and different inclusion (or ribbon) dimensions are fabricated and tested to investigate the effect of those parameters on the corrugation amplitude. The experimental results are compared with numerical analysis results and the mechanics of corrugation generation is discussed.

\section{Experiment}

\subsection{Design}

The geometry and working principle of a specimen composed of an elastomeric film and hard ribbons is shown schematically in Fig. 1. Following the convention of coordinate axes defined in Fig. 1, y-axis is the loading direction along which stretching is applied. In order to induce a uniform strain throughout the entire active region at the center and minimize the effect of gripping during tension test, the elastomeric film is machined to a dog-bone shape (Fig. 1) such that the two ends which are clamped during stretching are wider than the midsection. The central region of the dog-bone shaped specimen is narrow, with an area of $1 \times 1 \mathrm{~cm}^{2}$. To generate out-of-plane corrugation along $z$-axis, thin ribbons are placed on the surface of an elastomeric film, oriented in such a way that their long edges are parallel to the $\mathrm{x}$-axis. These ribbons are arrayed in a staggered manner, i.e., the first ribbon is located on the top surface of the elastomeric film, whereas the second ribbon sits on the bottom surface with proper inplane spacing to the first ribbon. The third ribbon is again located on the top surface whereas the fourth one sits on the bottom surface, etc. To accommodate Poisson's effect

A

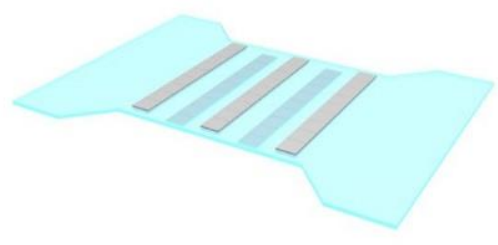

B

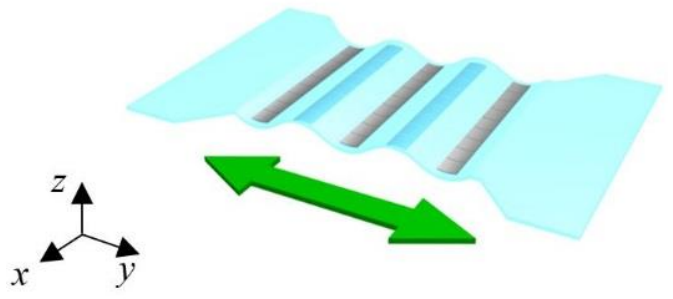

Fig. 1. Illustration of a composite specimen at stretch-free state (A) and $20 \%$ stretching state (B). The green arrow in (B) shows the direction of applied uniaxial tension. 
induced by the stretching along $y$-direction, each ribbon is broken into a 1D array of discrete platelets with width of $500 \mu \mathrm{m}$ and gap of $5 \mu \mathrm{m}$ along $x$-direction. Other geometric parameters used in this study are given in Table 1. Fixed parameters include the thickness of the film $(h=$ $200 \mu \mathrm{m})$ and the thickness of ribbons $(t=3 \mu \mathrm{m})$. Note that the wavelength of the corrugation is directly related to the period of the ribbon. For instance, at stretch-free state the wavelength is $2(L+s)$. In order to investigate the amplitude of corrugation under the same corrugation wavelength, the period of the ribbon is fixed at $1.3 \mathrm{~mm}$, whereas the ratio of ribbon spacing/length $(s / L)$ along $y$-direction varies between different samples for comparison (Table 1). Material-wise, polydimethylsiloxane (PDMS) and single crystal silicon are chosen for the elastomeric film and hard ribbons, respectively. The extremely large elastic moduli ratio between the two materials (the ratio of $\sim 10^{5}$ ) is beneficial in maximizing the corrugation amplitude [10]. To further investigate the effect of material elastic modulus mismatch on the amplitude of corrugation, PDMS with different mixing ratio $(3: 1,10: 1,15: 1)$ is used since different mixing ratio results in different elastic modulus as indicated in Table 1.

When a homogeneous elastomeric film is stretched, its length increases while maintaining a flat and an even surface profile. However, a heterogeneous film composed of an elastomeric film and hard ribbons develops a corrugation pattern upon stretching, as shown in Fig. 1(B), which is formerly described [10]. The difference between homogeneous (i.e., soft film only) and heterogeneous (i.e., soft film and hard ribbons) films mainly lies in the difference of their neutral axes. The axis for any homogeneous film coincides with its geometric center of interior section. Thus, uniform loading causes only stretching. In a heterogeneous film, however, the axis shifts towards an off-centered ribbon because the ribbon is much stiffer than the film. The staggered arrangement of ribbons results in a periodic shifting of axes. Thus, while uniform loading is applied to a heterogeneous film, internal bending moments is induced on the film. More details on the corrugation formation mechanism will be discussed in section 4 .

Table 1 - Design Parameters of the Composite Film

\begin{tabular}{|c|c|c|c|c|}
\hline & $>n$ & $\begin{array}{c}\leftrightarrow \\
\mathrm{s} \\
=200 \mu \mathrm{m}\end{array}$ & $3 \mu \mathrm{m}$ & \\
\hline $\begin{array}{c}\text { Ribbon } \\
\text { material }\end{array}$ & $\begin{array}{c}\mathbf{s} / \mathbf{L} \\
(\mu \mathrm{m} / \mu \mathrm{m})\end{array}$ & $\begin{array}{c}\text { Film } \\
\text { material }\end{array}$ & $\begin{array}{c}\text { PDMS } \\
\text { mixing } \\
\text { ratio }\end{array}$ & $\begin{array}{l}\text { PDMS } \\
\text { Young's } \\
\text { Modulus } \\
{\text { (kPa })^{[15]}}\end{array}$ \\
\hline Silicon & $150 / 500$ & PDMS & $10: 1$ & 750 \\
\hline Silicon & $250 / 400$ & PDMS & $10: 1$ & 750 \\
\hline Silicon & $350 / 300$ & PDMS & $10: 1$ & 750 \\
\hline Silicon & $150 / 500$ & PDMS & $3: 1$ & 868 \\
\hline Silicon & $150 / 500$ & PDMS & $15: 1$ & 360 \\
\hline
\end{tabular}

A
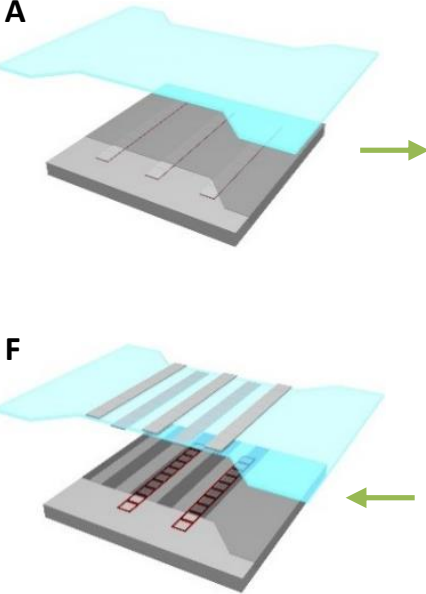

B

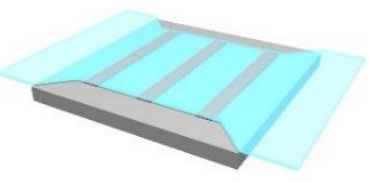

E

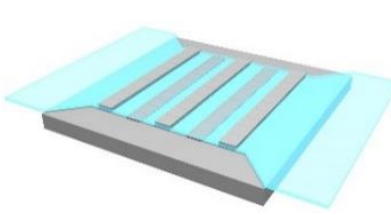

C
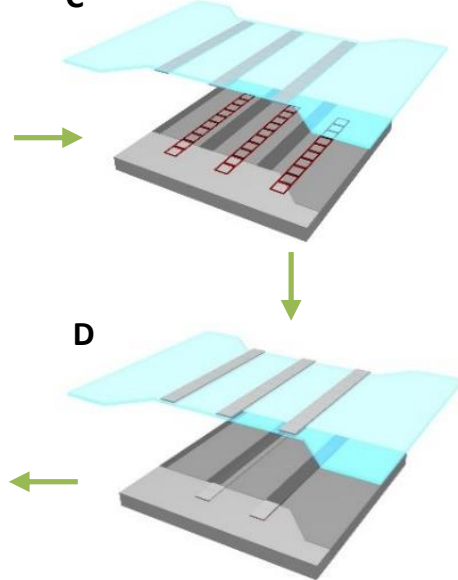

D

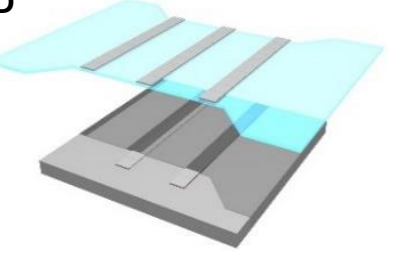

Fig. 2. Transfer printing process to form the composite film. (A) A PDMS film and the first silicon donor substrate are shown at separation state. (B) Contact is made between the PDMS film and silicon inks. (C) Rapid retrieval of the PDMS film delaminate the silicon inks from the first donor substrate such that the PDMS film is inked on one side. (D) The inked PDMS film is flipped over and delivered atop the second silicon donor substrate. (E) Contact is made between the non-inked side of PDMS film and the second silicon donor substrate with proper alignment. (F) Rapid retrieval of the PDMS film delaminates the silicon inks from the second donor substrate. The PDMS film is then inked on both sides, with proper in-plane spacing between the top and bottom silicon inks. 


\subsection{Fabrication}

The fabrication procedure is divided into three parts: preparation of silicon donor substrates, preparation of dogbone shaped PDMS films and transfer printing of silicon ribbons (or 1D array of silicon inks, under the context of transfer printing) onto a PDMS film. A donor substrate which contains silicon inks is made using standard microfabrication which is described as follows. First, one starts with a silicon-on-insulator wafer (SOI wafer, Ultrasil Corporation, USA) with 3- $\mu \mathrm{m}$-thick device layer (i.e., top silicon layer of a SOI wafer) and 1.1- $\mu \mathrm{m}$-thick buried oxide (BOX) layer. The device layer is patterned into the shape of inks which form ribbons by photolithography followed by deep reactive-ion etching (DRIE) until BOX layer is exposed. The exposed BOX layer is removed by $49 \%$ hydrofluoric acid (HF) solution and $\sim 1 \mu \mathrm{m}$ undercut is created around the rim of silicon. Spin coating of photoresist and flood exposure result in a rim of photoresist anchor which sits underneath the rim of each silicon ink. Immersing the donor into $49 \%$ HF for 8 hours to remove the BOX layer completes the preparation of a silicon donor substrate. Each silicon ink of a ribbon is now suspended while still weakly tethered to the handle layer by the photoresist anchor. Detailed description of this process can be found elsewhere [16]. Each composite specimen needs two donor substrates which provide the silicon ribbons on both side of the specimen.

The PDMS film is manufactured by a modified polymer molding process. A $200-\mu \mathrm{m}$-deep mold with a dog-bone shaped trench is made using a SOI wafer with $200-\mu \mathrm{m}$ thick device layer by photolithography and DRIE. The mold is then treated with trichloro- $(1 \mathrm{H}, 1 \mathrm{H}, 2 \mathrm{H}, 2 \mathrm{H}$ perfluorooctyl) silane (Sigma-Aldrich, USA) under vacuum for 1 hour to produce a non-stick monolayer on the silicon surface. PDMS base and curing agent (Sylgard 184, Dow Corning, USA) are mixed and degassed in vacuum for 1 hour before casting onto the mold. The mold, covered by PDMS precursor, is scraped by a razor blade to remove the excessive PDMS on the surface such that precursor can only stay inside the trench. The PDMS is then partially cured by heating up the mold on a $60^{\circ} \mathrm{C}$ hotplate for $30 \mathrm{~min}$ before the PDMS film is peeled from the mold.

Once silicon inks and PDMS films are prepared, transfer printing processes are performed to incorporate the silicon inks onto the PDMS film, as shown in Fig. 2. The partially cured PDMS film acts as a stamp during the whole transfer printing process. To integrate the first array of silicon inks, conformal contact is made between the first silicon donor substrate and the PDMS film. Fast retrieval of the PDMS film results in the delamination of silicon inks from their donor substrate. The inked PDMS film is then flipped over and delivered to the second donor substrate. Contact is made between the non-inked side of the PDMS film and the second donor substrate with proper alignment. The
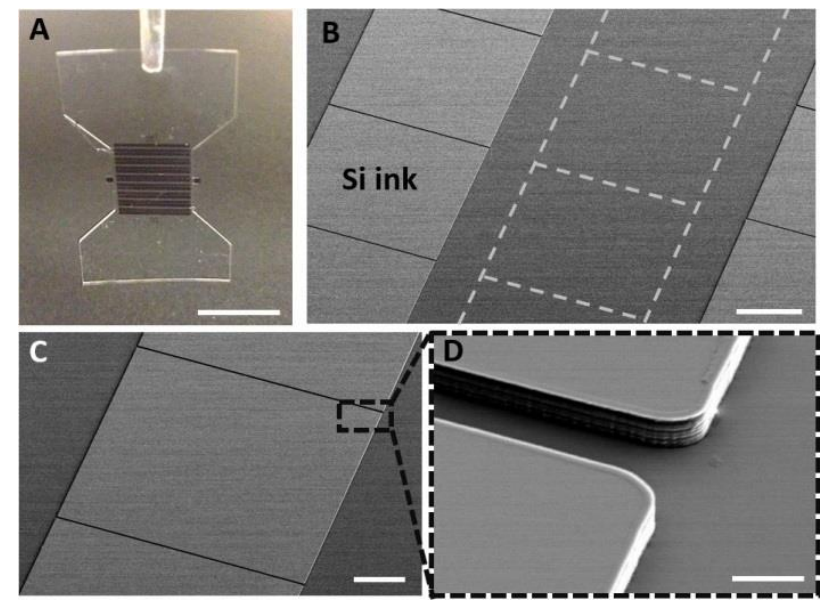

Fig. 3. Images of a fabricated specimen. (A) Photograph of a dog-bone shaped specimen for stretching test. Scale bar represents $1 \mathrm{~cm}$. (B) Scanning electron microscopy (SEM) image of the specimen surface. The dashed lines show the position of inks on the back side. Scale bar represents $200 \mu \mathrm{m}$. (C) Magnified SEM image. Scale bar represents $100 \mu \mathrm{m}$. (D) Further magnified SEM image showing the contact between silicon inks and PDMS. Scale bar represents $5 \mu \mathrm{m}$.

PDMS film is rapidly retrieved and the second array of silicon inks is grabbed off the donor substrate, similar to the first transfer printing. For simple and accurate alignment between the top and bottom silicon pattern, two types of ink layout are made corresponding to the first and second silicon patterns, which eventually correspond to the top and bottom silicon inks on the final composite film. Both types of donor substrate contain an array of silicon inks for corrugation formation as well as two alignment inks for alignment purpose. The two types have identical silicon arrays but the location of alignment inks on the second donor is shifted by a distance of $(L+s)$ along $y$ direction. Therefore, during the second transfer printing, proper spacing between the first and second silicon ink arrays is achieved as long as the alignment inks on the second donor substrate is aligned to the alignment inks on the PDMS film which are picked up earlier during the first transfer printing. After the transfer printing processes, the composite film is annealed inside a $70^{\circ} \mathrm{C}$ convection oven for 10 hours to fully cure the PDMS film, which is only partially cured before the transfer printing. During this annealing process, covalent bonding is formed between silicon and PDMS as a result of the hydroxyl condensation reaction [17]. The strong bonding between the silicon inks and PDMS film is important to ensure that no interfacial delamination occurs during stretching. The optical and scanning electron microscopy (SEM) images of a fabricated specimen are shown in Fig. 3.

\subsection{Characterization}



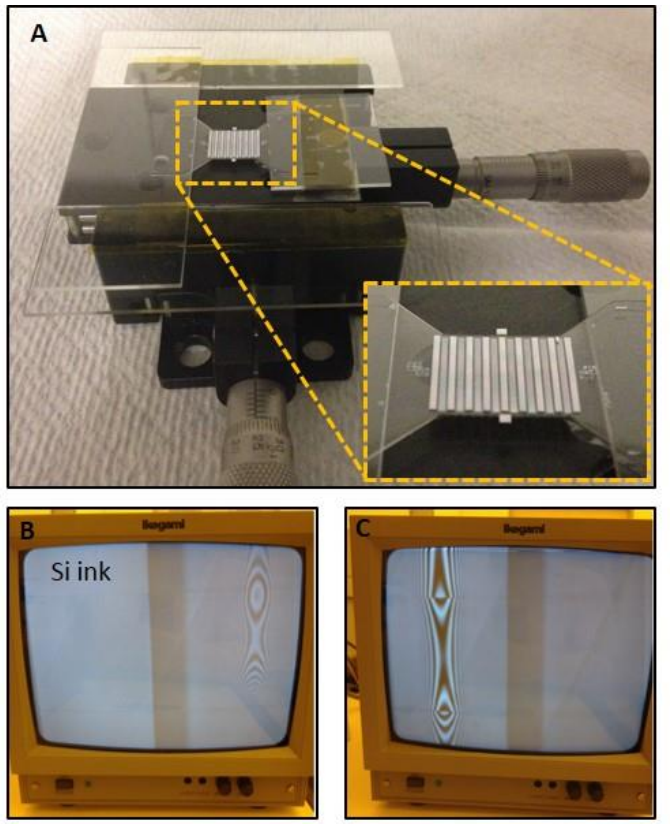

D Focal plane

E

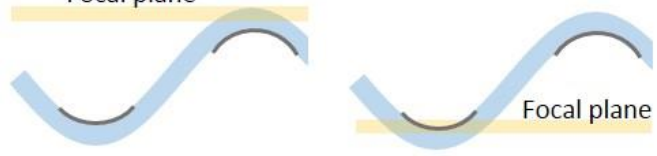

Fig. 4. Setups for corrugation characterization. (A) A custom-built mechanical stretcher with a mounted specimen. (B-E) Optical profiling for noncontact 3D surface topography measurements using white light interferometry. (B) Interference fringes when the profiler focuses on the corrugation crest of the sample. (C) Interference fringes when the profiler focuses on the corrugation trough of the sample. (D, E) Side view illustration of the focal plane position corresponds to (B) and $(\mathrm{C})$, respectively.

The corrugation profile of the fabricated specimens are characterized in situ using a custom made mechanical stage (Fig. 4(A)) and optical profiling (Fig. 4(B-E)). To maintain the stretch-free state of specimens during handling, all specimens are attached to a plastic sheet before they are mounted on the stretcher. The inset of Fig. 4(A) shows a specimen at a stretch free state. For qualitative observation of corrugation, the sample is stretched under the objective lens of an optical microscope. For quantitative measurement of corrugation amplitude and wavelength, an optical profiler (Wyko NT1000) is used and the procedure is illustrated in Fig. 4(B-E). The optical profiler is a vertical scanning interferometer using white light source. The incoming white light is divided into two beams by a beam splitter, one is sent to the corrugated sample surface and the other to a reference mirror. When the reflected light from these two surfaces recombines, an interference pattern (fringing pattern) forms onto the detector. Maximum contrast of fringes is achieved at the location where the

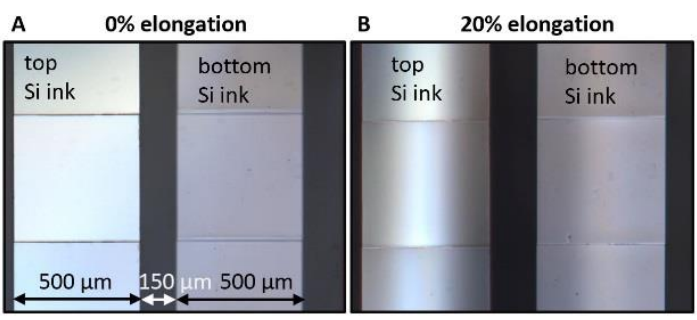

Fig. 5. Optical microscopic images of a specimen upon stretching. (A) $0 \%$ stretching state. (B) $20 \%$ stretching state. A darkness gradient appears on both the top and bottom silicon inks upon stretching which indicates the corrugated surface profile. In addition, the bottom silicon inks come into focus as a result of corrugation.

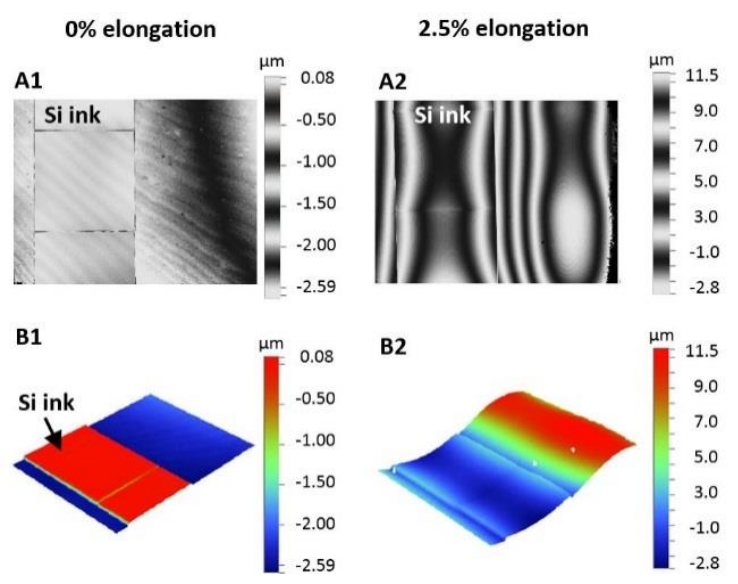

Fig. 6. Optical profiler measurement results (A) measured $2 \mathrm{D}$ contour of a specimen under $0 \%$ and $2.5 \%$ stretching. Different fringes represent different height along out-ofplane axis and their density indicates the slope. (B) Reconstructed 3D surface profile from the measured height data.

sample height overlaps with the focal plane of the instruments. Fig. 4 (B, C) demonstrate how the fringes evolve as the focal plane moves. During profiling, the reference mirror is scanned vertically such that each point on the sample surface passes through the focus. Fig. 4 (D, E) illustrate how the focal plane is scanned from the crest to the trough of the corrugated specimen.

To study the corrugation development numerically, we use the commercial finite element software ABAQUS. We consider the mid cross section of the thin film, i.e. $y-z$ plane, and model it as a $2 \mathrm{D}$ elastic continuum. A full $3 \mathrm{D}$ model will be computationally prohibitive and thus we consider the two limiting cases of plane strain (PE) and plane stress (PS). The PDMS is modelled as a NeoHookean hyperelastic material and its material properties (e.g. initial Young's modulus and Poisson's ratio) are taken as per the experimental measurements [15]. Silicon is modelled as linear elastic material. Perfect bonding is assumed at the PDMS/silicon interface. Eight node 
biquadratic finite elements (Q8) is used throughout entire domain.

\section{Results}

Fig. 5 shows the optical microscopic images of the soft composite film under stretching. Up to $20 \%$ stretching is applied continuously to the sample and the image corresponding to $0 \%$ and $20 \%$ stretching is shown in Fig. 5(A) and Fig. 5(B), respectively. Corrugation can be qualitatively observed by comparing the contrast of the two images. Under $0 \%$ stretching, both the top and bottom silicon appear to be flat since their color is almost uniform. The bottom silicon is darker than the top silicon due to light scattering from PDMS layer. As 20\% stretching is applied, a darkness gradient across the silicon along the loading axis can be easily observed. This can be attributed to the curvature of the silicon ribbons, generated by the bending moment. The central part of the silicon appears to be brighter because this region remains to be relatively flat so the incident light can still be reflected back to the camera. However, close to the edge of silicon ribbons the slope becomes larger such that the incident light starts to get deflected, leading to darker color. Another observation is that the bottom silicon ink starts to come into the depth of focus as stretching is applied. This observation further confirms the corrugation formation upon stretching.

The corrugation pattern is also quantitatively measured in situ by an optical profiler, and the results are shown in Fig. 6 and Fig. 7. After the vertical scan through the sample surface, the surface contour is obtained with the results shown in Fig 6(A1-A2). Each fringe on the contour represents a certain height, therefore the density of fringes indicates the slope around that area. Moreover, the 3D topography is reconstructed, shown in Fig. 6(B1-B2). It can be seen that a periodic corrugation pattern is developed as the stretching is applied.

A
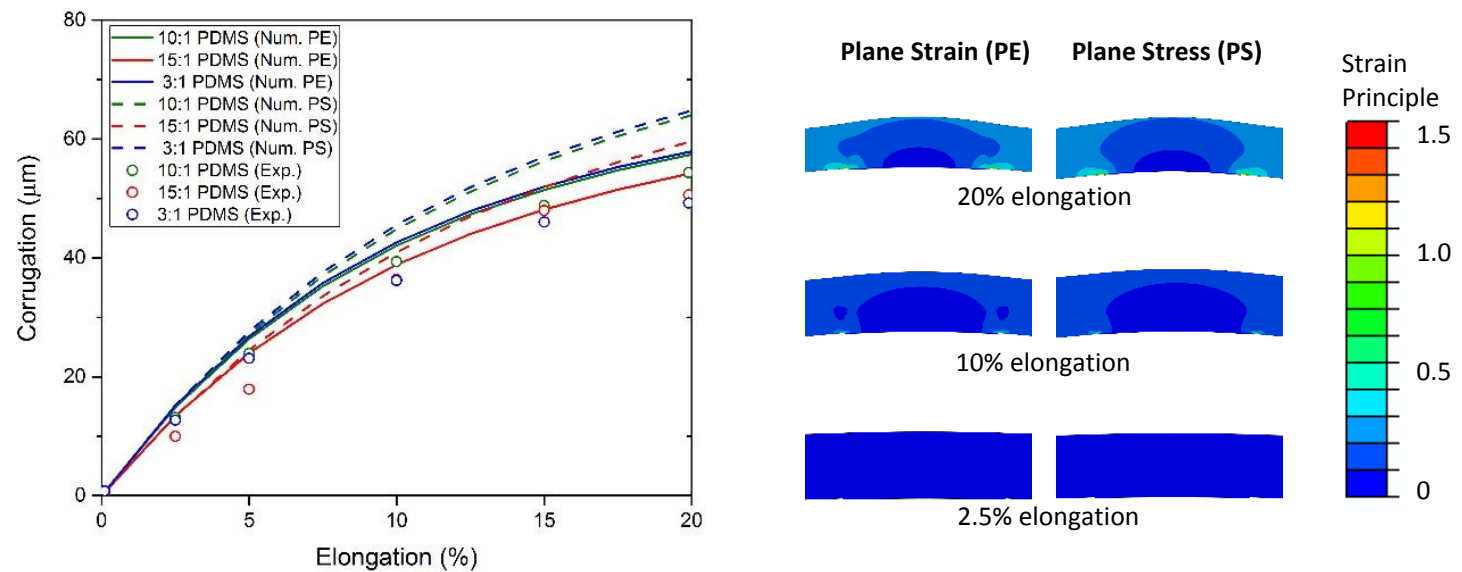

B
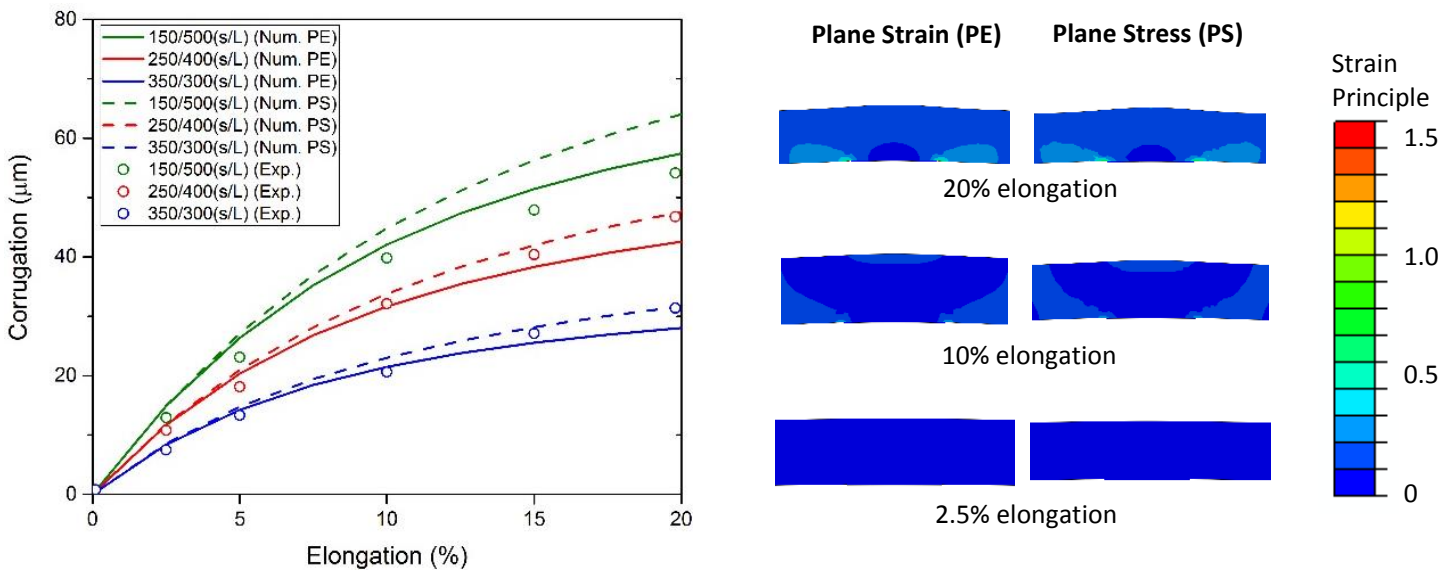

Fig. 7. Corrugation development as a function of elongation for samples with different PDMS mixing ratio (A) or different $\mathrm{Si}$ spacing/length ratio (B). Hollow circles represent experimentally measured results, whereas solid and dashed curves represent numerical models under plane strain (PE) and plane stress (PS) assumptions, respectively. Successive graphs on right show the numerical results for the strain fields as well as deformed shape of the films under $2.5 \%, 10 \%$ and $20 \%$ of elongation. The graphs next to plot (A) correspond to $500 \mu \mathrm{m}$ silicon ribbon, whereas those next to (B) correspond to $300 \mu \mathrm{m}$ silicon ribbon. 
The development of corrugation as a function of applied elongation is shown in Fig. 7. Corrugation amplitude is measured as the vertical distance between the crest and trough of the sinusoidal corrugation pattern and plotted as hollow circles. In addition to the sample with optimized design parameter (10:1 PDMS, $500 \mu \mathrm{m}$ silicon and $150 \mu \mathrm{m}$ spacing), specimens with other parameters as listed in Table 1 are also tested and the results are summarized in Fig. 7. From Fig. 7(A), it can be seen that the corrugation amplitude is not sensitive to the modulus change of PDMS film. For example, corrugation amplitude of $\sim 50 \mu \mathrm{m}$ is observed at $20 \%$ stretching regardless of the mixing ratio of the PDMS film. The effect of spacing is also investigated by maintaining the period of corrugation and changing the fraction of spacing. Specifically, the spacing/length ratio $(s / L)$ is varied from 0.3 to 1.2 following Table 1 . As the fraction of spacing increases, the corrugation amplitude decreases (Fig. 7(B)).

The curves in Fig. 7 represent the computational models for specimens with different PDMS mixing ratios (Fig. 7(A)) and different silicon ink spacing (Fig. 7(B)). Solid and dashed curves represent the numerical model under the assumption of plane strain and plain stress, respectively. Under the same geometric and material parameters, the plane stress model predicts a larger corrugation amplitude compared to the plane strain model. Compared to the experimental results, the numerical model reproduce, qualitatively and to some extent quantitatively (e.g. red and blue curves in Fig. 7(B)), the same pattern of corrugation development as seen experimentally (Fig. 7). For example, both the computational and experimental results reveal that the corrugation increase rapidly under small values of stretching but start to saturate as larger elongation is applied. Nevertheless some quantitative deviations are observed and the reason is discussed in the next section.

\section{Discussion}

The development of corrugation is explained by the transformation theory of composites. Fig. 8 shows the cross section of a homogeneous and heterogamous film under stretch free state. The center line and neutral axis are obtained by transformation rule and their locations are

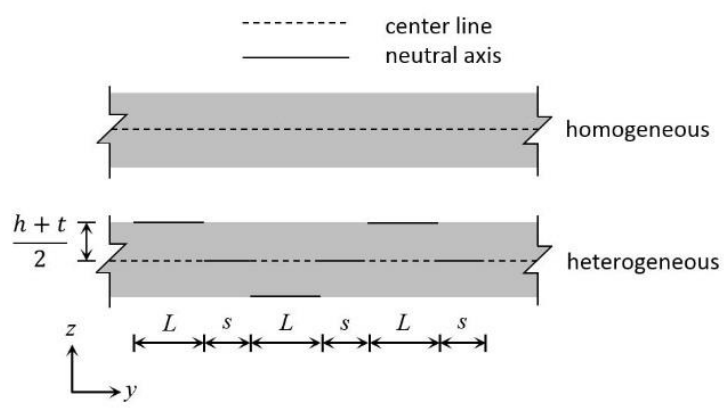

Fig. 8. Location of center lines and neutral axes of homogeneous and heterogeneous film. In the homogeneous case, the neutral axis coincides with the neutral axis. illustrated in Fig. 8. Both homogeneous and heterogeneous films are illustrated here with identical thickness, since the thickness of a silicon ribbon is only $1.5 \%$ of the PDMS and is considered as negligible. For the portion with pure PDMS, the neutral axis coincides with the center of area of the PDMS section. For the portion where silicon is attached to PDMS, the neutral axis is computed as the centre of area of the transformed section (i.e. the equivalent homogenized section where the silicon area is transformed into an equivalent PDMS area). The distance between the position of the neutral axis and the center line is then given by:

$$
d=\frac{E_{S i} t}{E_{S i} t+E_{P D M S} h}\left(\frac{h+t}{2}\right)=\frac{r}{r+h / t}\left(\frac{h+t}{2}\right) \cong \frac{h+t}{2}
$$

Since the material modulus contrast $\mathrm{r}$, define as $E_{S i} / E_{P D M S}$, is very large $\left(\sim 10^{5}\right)$, the axis is almost coincident with the center line of silicon ink, at a distance of $(h+t) / 2$ from center of the PDMS section.

Several parameters including silicon/PDMS modulus contrast $\left(E_{s i} / E_{P D M S}\right)$ and spacing between silicon ribbons $(s)$ affect the corrugation amplitude as shown by the previous numerical study [10]. To understand the effect of these two parameters on the corrugation amplitude under a fixed stretch (elongation $=20 \%$ ), the corrugation amplitude upon changing these parameters is numerically investigated and the results are presented as a contour plot (Fig. 9). The dots in the plot correspond to combination between $E_{S i} / E_{P D M S}$ and $s$ studied in the experiments.

Effect of spacing between silicon ribbons: Numerical simulation for specimens with different normalized spacing $s / h$ is conducted. To be consistent with the experimental procedure, the sum of the silicon length and the silicon spacing, i.e., $(s+L)$, is set as a constant. Therefore the wavelength of corrugation remains to be the same as the spacing varies. It is found from the numerical study that increasing the spacing leads to a decreased corrugation amplitude (Fig. 9). This may be explained as follows. As the spacing increases, the length of the silicon ink decreases. Since the ink thickness is kept constant, a shorter ink possesses a higher bending stiffness $\left(\propto L^{-3}\right)$. Thus the ink resists bending deformations more as it gets shorter and prevent further development of corrugation a.

Effect of material modulus contrast: Previous numerical study [10] predicts a positive correlation between the material modulus contrast and the corrugation amplitude. However, it is experimentally observed that varying the mixing ratio of PDMS (thus the modulus of PDMS) does not affect the corrugation amplitude significantly (Fig. 7(A)). This is due to the fact that the contrast of modulus between silicon and PDMS remains to be quite high $\left(\sim 10^{5}\right)$ regardless of the mixing ratio of PDMS. Nevertheless, numerical analysis conducted to investigate the effect of material modulus contrast over a wider range $\left(1-10^{6}\right)$ confirms that positive correlation between corrugation amplitude and modulus contrast holds up to a modulus ratio of $10^{4}$. Beyond that the effect is saturated and might even be slightly reversed eventually, as 
shown by the almost vertical striation of the contours in Fig 9. This phenomenon can be explained as follows. At a lower modulus ratio, as the modulus contrast increases the eccentricity of neutral axis is enhanced. However at a very high modulus ratio $\left(\sim 10^{5}\right.$ in our experiment), the location of the neutral axis may be assumed to always lie within the silicon ribbons to an excellent approximation. Therefore the internal eccentricity and the resulting bending moment have no room to increase anymore as the material modulus contrast increases. However, lower modulus of PDMS allows more deformation of PDMS. Thus, an increased portion of the deformation is accommodated through stretching and lateral contraction of a PDMS film rather than through increasing its curvature. This causes slightly reduction of corrugation amplitude. Nonetheless, the overall corrugation is dominated by the eccentricity of neutral axes which saturates under large material modulus contrast, and leads to the almost vertical contour lines in Fig. 9.

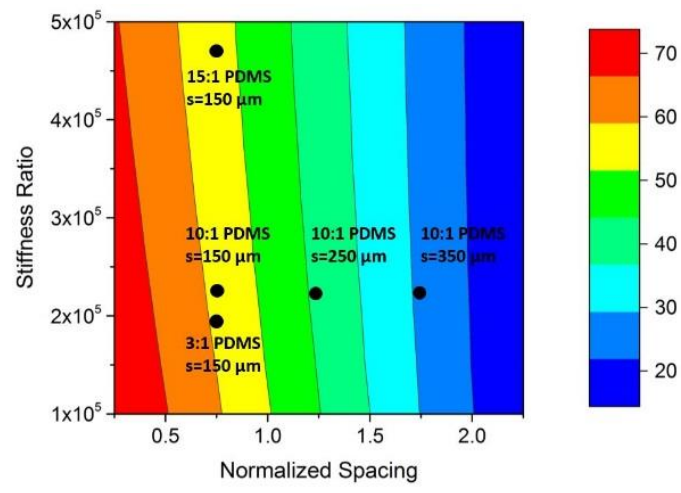

Fig. 9. Contour plot of corrugation (at $20 \%$ elongation) as a function of material stiffness contrast and normalized silicon spacing. Dots correspond to the parameters used in experiment.

As shown in Fig.7 the experimental data matches numerical prediction to a good extent but some deviations are also observed. Some quantitative errors can be attributed to the errors caused by the fabrication and testing procedure. Although numerical modeling uses exact geometrical dimensions, the actual specimens may have slightly different geometries caused by fabrication errors. For example, the size of silicon ribbon and PDMS film may not be exactly the same as what are listed in Table 1. Also the actual spacing between silicon ribbons is affected by the possible misalignment during transfer printing step, which has a tolerance of about $5 \mu \mathrm{m}$. In addition, the actual loading axis may not be completely aligned with the $y$-axis during tension test.

Furthermore, silicon inks and a PDMS film may not form perfect bonding in between, unlike what is assumed in the numerical modeling. While no interfacial separation has been observed during the experiments, it is still possible that some interfacial deformation may be present.
Deviations from perfect bonding may be responsible for some discrepancies between the numerical and experimental observations in the limit of large elongation especially for the case with low PDMS mixing ratio. Our preliminary work suggests that accounting for the interfacial deformations between silicon inks and a PDMS film leads to a decrease in the predicted corrugation amplitude and thus brings the numerical calculations closer to the experimental measurements in the case with low PDMS mixing ratio (Fig. 7A). Future work will study this effect in further details and will account for the interfacial response by modeling an interfacial region between silicon inks and a PDMS film. Properties of the interface will be taken from relevant experimental measurements.

Finally, either plane strain or plane stress conditions are assumed in the numerical model to approximate the corrugation development in the composite film. While the planar numerical simulations of the $y-z$ plane approximate the experimental measurements to a good extent, it should be noted that the actual film has comparable dimensions in the $\mathrm{x}-\mathrm{y}$ plane. Consequently, effects such as the nonlinear deformation due to the compression along $x$-direction, or other boundary effects due to sample clamping or dog-bone geometry, might not be fully captured by the numerical model. Also, there exist small gaps between individual silicon inks in each 1D array along $x$-direction, which makes the scenario more complicated. A full 3D model for future work would be capable of capturing these effects. In such a model, mesh sizes of sub-micron scale must be used to resolve the inter-ink gaps as well as the ink thickness. For a sample size on the centimeter scale, this will require more than a billion elements. Innovations in nonconforming meshes or adaptive mesh refinement are required to overcome this computational challenges. This is a subject of further research.

\section{Conclusion}

In this paper, we apply the emerging transfer printing technique to realize a soft composite film with the novel capability of achieving tunable surface topography under uniaxial tension. The fabricated composited film consists of a PDMS film and an array of transfer printed silicon ribbons with staggered distribution. We experimentally demonstrated that such a composite film develops corrugated surface profile upon uniaxial tension. The spatial distribution of silicon ribbons are crucial to the corrugation formation, whereas the transfer printing processes developed in this work ensures the required spatial organization. The corrugation pattern in response to tension is characterized in both qualitative and quantitative manners using optical methods. The characterization results show that a periodic wavy surface topography is developed spontaneously upon stretching, whereas the amplitude depends on the extent of stretching unlike in buckling-induced corrugations explored previously. We envision that future extension of these findings may lead to applications such as tunable surface with controllable dry adhesion and wettability, mechanical tunable metamaterials 
and novel electronic devices in an unusual form. In addition, overlaying multiply layers of such composite films in an aligned manner will result in a bulk material with tunable void sizes and shapes, which can leads to potential applications such as devices for water filtering and tunable acoustic bandgaps.

\section{References}

[1] P. Lin, S. Vajpayee, A. Jagota, C. Huid. S. Yang, Mechanically tunable dry adhesive from wrinkled elastomers. Soft Matter, 4, (2008) 1830 Sinusoidal phase grating created by a tunably buckled surface 1835

[2] H. Jeong, M. Kwak, K. Suh, Stretchable, Adhesion-tunable dry adhesive by surface wrinkling, Langmuir, 26 (2010) 2223 Sinusoidal phase grating created by a tunably buckled surface 2226

[3] J. Chung, J. Youngbloodb, C. Stafford, Anisotropic wetting on tunable micro-wrinkled surfaces, Soft Matter, 3 (2007) 1163-1169

[4] S. Xu, Z. Yan, K.-I Jang, W. Huang, H. Fu, J. Kim, et al., Assembly of micro/nanomaterials into complex, three-dimensional architectures by compressive buckling, Science, 347 (2015) 154-159.

[5] D. Khang, H. Jiang, Y. Huang, J.A. Rogers, Stretchable form of single-crystal silicon for high-performance electronics on rubber substrates, Science, 311 (2006) 208-212

[6] C. Harrison, C. Stafford, W. Zhang, A. Karim, Sinusoidal phase grating created by a tunably buckled surface, Appl. Phys. Lett. 85 (2004) 4016

[7] E. P. Chan, A. J. Crosby, Fabricating microlens arrays by surface wrinkling, Adv. Mater. 18, (2006) 3238-3242

[8] S. Lee, S. Kim, T. Kim, Y. Kim, M. Choi, S. Lee, et al., Reversibly stretchable and tunable terahertz metamaterials with wrinkled layouts, Adv. Mater., 24 (2012) 3491-3497

[9] S.Yang, K. Khare, P. Lin, Harnessing Surface Wrinkle Patterns in Soft Matter, Adv. Funct. Mater., 20 (2010) 2550-2564
[10] Q. Chen, A. Elbanna, Tension-induced tunable corrugation in twophase soft composites: Mechanisms and implications, Extreme Mechanics Letters 4 (2015) 26-37

[11] M.A. Meitl, Z. Zhu, V. Kumar, K. Lee, X. Feng,Y. Huang, et al., Transfer printing by kinetic control of adhesion to an elastomeric stamp, Nature. Mater., 5 (2006) 33-38

[12] S. Kim, J. Wu, A. Carlson, S. Jina, A. Kovalskya, P. Glass,et al., Microstructured elastomeric surfaces with reversible adhesion and examples of their use in deterministic assembly by transfer printing, Proc. Natl. Acad. Sci., 107 (2010) 17095-17100

[13] A. Carlson, A. M. Bowen, Y. Huang, R. G. Nuzzo, J.A. Rogers, Transfer printing techniques for materials assembly and micro/nanodevice fabrication, Adv. Mater., 24 (2012) 5284-5318

[14] X. Feng, M.A. Meitl, A.M. Bowen, Y. Huang, R.G. Nuzzo and J.A. Rogers, Competing Fracture in Kinetically Controlled Transfer Printing, Langmuir, 23 (2007) 12555-12560

[15] D. Armani, C. Liu, and N. Aluru, Re-configurable fluid circuits by PDMS elastomer micromachining, Proc. IEEE MEMS, (1999) 222227.

[16] H. Keum, A. Carlson, H. Ning, A. Mihi, J. Eisenhaure, P.V. Braun, J.A. Rogers, and S. Kim, Silicon Micro-Masonry Using Elastomeric Stamps for Three-Dimensional Microfabrication, J. Micromech. Microeng., 22 (2012) 055018

[17] S. Kim, Y. Su, A. Mihi, S. Lee, Z. Liu, T.K. Bhandakkar, J. Wu, J.B. Geddes III, H.T. Johnson, Y. Zhang, J.-K. Park, P.V. Braun, Y. Huang, and J.A. Rogers, Imbricate scales as a design construct for microsystems technologies, Small, 8 (2012) 901-906. 